\title{
SOME EXTENSIONS OF THE LANCZOS-ORTIZ THEORY OF CANONICAL POLYNOMIALS IN THE TAU METHOD
}

\author{
M. E. FROES BUNCHAFT
}

\begin{abstract}
Lanczos and Ortiz placed the canonical polynomials (c.p.'s) in a central position in the Tau Method. In addition, Ortiz devised a recursive process for determining c.p.'s consisting of a generating formula and a complementary algorithm coupled to the formula. In this paper

a) We extend the theory so as to include in the formalism also the ordinary linear differential operators with polynomial coefficients $D$ with negative height

$$
h=\max _{n \in N}\left\{m_{n}-n\right\}<0,
$$

where $m_{n}$ denotes the degree of $D x^{n}$.

b) We establish a basic classification of the c.p.'s $Q_{m}(x)$ and their orders $m \in M$, as primary or derived, depending, respectively, on whether $\exists n \in$ $\mathbf{N}: m_{n}=m$ or such $n$ does not exist; and we state a classification of the indices $n \in \mathbf{N}$, as generic $\left(m_{n}=n+h\right)$, singular $\left(m_{n}<n+h\right)$, and indefinite $\left(D x^{n} \equiv 0\right)$. Then a formula which gives the set of primary orders is proved.

c) In the rather frequent case in which all c.p.'s are primary, we establish, for differential operators $D$ with any height $h$, a recurrency formula which generates bases of the polynomial space and their multiple c.p.'s arising from distinct $x^{n}, n \in N$, so that no complementary algorithmic construction is needed; the (primary) c.p.'s so produced are classified as generic or singular, depending on the index $n$.

d) We establish the general properties of the multiplicity relations of the primary c.p.'s and of their associated indices.

It becomes clear that Ortiz's formula generates, for $h \geq 0$, the generic c.p.'s in terms of the singular and derived c.p.'s, while singular and derived c.p.'s and the multiples of distinct indices are constructed by the algorithm.
\end{abstract}

\section{INTRODUCTION}

Lanczos and Ortiz's Tau Method ([1]-[5]) is founded on the determination of numerical solutions of linear ordinary differential equations with polynomial coefficients $p(x)$

$$
D y(x)=\sum_{i=0}^{v} p_{i}(x) \frac{d^{i}}{d x^{i}} y(x)=0,
$$

subjected to given supplementary (initial value, boundary value) conditions.

Received by the editor May 23, 1995 and, in revised form, April 12, 1996.

1991 Mathematics Subject Classification. Primary 65L05, 65L10, 65D99.

Key words and phrases. Initial value problems, boundary value problems, ordinary differential equations, approximation of functions, Tau Method, Lanczos-Ortiz's canonical polynomials.

This paper is a modified version of part of the author's thesis at Pontifícia Universidade Católica, Departamento de Informática, PUC-Rio. 
The essential idea that makes the method notable is the fact that, instead of looking for a polynomial approximate solution truncating an infinite power series expansion, it searches for an exact polynomial solution $y_{n}^{*}(x)$ of a perturbed equation of the given equation.

This exact polynomial solution is constructed by adding to the second member of the given equation a polynomial term $H_{n}(x)$ of degree $n$, which is a perturbative term (and thus contains an $n$-th order approximant to the zero polynomial):

$$
D y_{n}^{*}(x)=H_{n}(x)
$$

This way the exact solution of the system thus perturbed will be a polynomial, which should also exactly satisfy the same supplementary conditions of the given problem. Consequently $y_{n}^{*}(x)$ is an $n$-th order polynomial approximant to the exact solution $y(x)$ of the given problem.

Starting from this elementary basis and this central characteristic idea, the method diversifies and widens its comprehensiveness far beyond this initial formulation: the differential operator may have as coefficients any smooth functions approximated with arbitrary precision by rational functions, possibly by the Tau Method itself; the differential equations may be nonhomogeneous, nonlinear, functional, or partial-derivation equations, or even a system of differential equations $([6]-[15])$.

The comprehensiveness of the method results largely from the varied forms that the perturbative polynomial $H_{n}(x)$ may assume, and also from the fact that the method allows a diversified choice of the basis $V=\left\{V_{n}(x), n \in \mathbf{N}\right\}$ (where $\mathbf{N}$ is the set of natural integers) of the vector space $P(x)$ of univariate polynomials, with respect to which the polynomials $H_{n}(x)$ and $y_{n}^{*}(x)$ will be expressed.

Incidentally, Lanczos and Ortiz have shown that, in addition to the canonical basis and other well-known general polynomial bases $V$ (Chebychev, Legendre, Hermite, etc.), it is possible to define in $P(x)$ a type of specific polynomial basis $Q=\left\{Q_{m}(x), m \in M \subseteq \mathbf{N}\right\}$, with $M$ properly defined, determined by each linear differential operator $D$ relative to $V$, which plays a central role in the Tau Method. Thus Ortiz developed a recursive approach for the determination of c.p.'s that can be employed in all the widely different applications of the method.

This recursive approach has the following characteristics:

a) the basic theory is not developed for differential operators $D$ whose action reduces the degree of the polynomials in $P(x)$ (that is, for $D$ with height $h<0$ );

b) the generating formula gives certain c.p.'s $Q_{m}(x)$ as a linear combination of some $x^{n}$ (being $n=m-h$ ) and the c.p.'s $Q_{r}(x), r<m$ (these c.p.'s will be herein identified as primary-generic in our classification stated below); the remaining c.p.'s are determined only in an algorithmic-constructive way, which breaks the recurrency of the formula.

In this paper we attempt to reanalyze and extend Lanczos and Ortiz's basic ideas concerning c.p.'s of the Tau Method:

a) We include in the entire theory the differential operators $D$ with negative height, that is, which reduce the degree of the polynomials in $P(x)$;

b) We establish a classification of the c.p.'s $Q_{m}(x)$ (and their orders $m$ ) as primary and derived and a classification of the indices $n \in \mathbf{N}$ as generic, singular and indefinite (see Definitions 1, 2, and 5); furthermore we prove a formula which gives the set of primary orders; 
c) We introduce the concept of index-multiplicity structure of $D$ (see Definition $4)$;

d) In the very frequent case in which all the c.p.'s are primary we prove a formula which, for differential operators $D$ with any height, generates an entire basis of c.p.'s $Q_{m n}(x)$ and also all their multiples of distinct indices $n$, in a formal-recurrent way, with no need for a complementary algorithmic construction; the (primary) c.p.'s $Q_{m n}(x)$ defined by this generating formula are then subclassified as generic and singular, depending on the c.p.'s index $n$;

e) We establish the general properties of the index-multiplicity structure and its general relations with the multiplicity of c.p.'s and with the possibility of existence of derived (i.e., nonprimary) c.p.'s;

The classification of c.p.'s and their orders, and the classification of the indices play a guiding role in the entire analysis.

The theoretical results here obtained also give support to the construction and control of algorithms for computer implementation.

A brief recall on the essential features of the Lanczos-Ortiz theory on c.p.'s is given in $\S 2$ below. In $\S 3$ we begin to introduce our new theoretical constructions, that is a), b) indicated above. In $\S 4$ is given $c)$, in $\S 5$ follows $d$ ). The proof of the recurrent generating formula is given in the Appendix, and in $\S 6$ is stated e). Some examples, worked on by our software GPC, are outlined in $\S 7$ and some further remarks, in $\S 8$, conclude the article.

\section{Revisiting Lanczos and ORTiz's CANONICAL POLYNOMIALS}

Ortiz and Llorente ([3], [4], [5]) have shown that an ordinary linear differential operator $D$ of finite order, with polynomial coefficients, acting in the vector space of univariate polynomials

$$
D: P(x) \rightarrow P(x),
$$

produces an image $D P(x)$ of finite co-dimension having as upper bound $v+h$, where $h$, called the height of $D$, is

$$
h=\left[\max _{n \in \mathbf{N}}\left(m_{n}-n\right)\right] \in \mathbf{N},
$$

where

$m_{n}$ - is the degree of the generating polynomial $D x^{n}$;

$\mathbf{N}$ - is the set of the natural integers;

The finiteness of the co-dimension of $D P(x)$ in $P(x)$ is equivalent to the following:

Lemma. There is a finite subset $S \subset \mathbf{N}$ of $\mathbf{N}$ such that $P(x)$, in the codomain, decomposes into a direct sum

$$
P(x)=D P(x) \oplus R(x),
$$

where basis;

a) $R(x)$ is generated by the elements of the subset $\left\{x^{s}, s \in S\right\}$ of the canonical

b) for every $m \in M=N-S$, there is in $D P(x)$ a polynomial $p_{m}(x)=x^{m}+$ $R_{m}(x), R_{m}(x) \in R(x)$, with degree smaller than $m$, and $P=\left\{p_{m}(x), m \in M\right\}$ is a basis of $D P(x)$; 
(This proposition is simply a restatement of Ortiz-Llorente's main result in [3].)

Ortiz has called $R(x)$ the space of residuals. Moreover the inverse image by $D$ of each $p_{m}(x) \in D P(x)$ is a polynomial $Q_{m}(x) \in P(x)$ called canonical polynomial of order $m$, or, more generally, is an infinite multiplicity (an equivalence class) of canonical polynomials $Q_{m}(x)+U_{D}(x)$ where $U_{D}(x)$ is an arbitrary polynomial in the polynomial kernel $U_{D}$ of $D$ in $P(x)$; clearly such polynomials, called multiple polynomials, differ themselves by a polynomial $U_{D}(x) \in U_{D}$.

Any infinite sequence $Q=\left\{Q_{m}(x), m \in M\right\}$ of c.p.'s of distinct orders constitutes a basis $Q$ of $P(x)$ called a basis of canonical polynomials of $D$ relative to the canonical basis $X$ : a basis $Q$ of $P(x)$ modulo $U_{D}$ is the inverse image by $D$ of the basis $P$ of $D P(x)$.

The class $\mathbf{D}$ of linear differential operators $D$ with polynomial coefficients and the class $\mathbf{L}$ of the sets $L$ of the multiple canonical polynomials $\left\{\left\{Q_{m}(x)+U_{D}(x)\right\}, m \in\right.$ $\left.M, U_{D}(x) \in U_{D}\right\}$ are in one-to-one correspondence.

Lanczos and Ortiz's c.p.'s play an essential role in the recursive approach of the Tau Method. For this very reason, improvements in the theory of c.p.'s may have significant implications for the wide range of applications of the method.

\section{Determination of the Set $M^{*} \subseteq M$ OF PRimary orders}

Let us now consider in the theory the linear differential operators $D$ with $h \in \mathbf{Z}$ ( $\mathbf{Z}$ being the set of all integers): it is clear that the previous lemma remains entirely valid, since for $h<0$, the co-dimension of $D P(x)$ in $P(x)$ has as upper bound $\nu$ and the finiteness of this co-dimension is equivalent to the statement of the previous lemma.

The linear differential operator $D$ is uniquely determined by its action on a basis of $P(x)$, in particular on the canonical basis $X-$ that is, by the set $\left\{D x^{n}, n \in \mathbf{N}\right\}$ of generating polynomials of $D$.

Then the action of $D$ in $X$ induces the application "degree of $D x$ " denoted by $m$ :

$$
m: N^{*} \rightarrow M: n \mapsto m_{n}
$$

(when the context allows no ambiguity, we may, by an abuse of language, denote also by $m$ the degree of any polynomial in $D P(x)$, particularly the degree $m_{n}$ ).

Let us then introduce the following definitions:

\section{Definition 1.}

$F=\left\{n: m_{n}=n+h, n \in \mathbf{N}, n \geq-h\right\}$ - the set of D-generic indices,

$G_{d}=\left\{n=m_{n} \neq n+h, n \in \mathbf{N}, n>-h\right\}-$ the set of D-singular indices,

$G_{i n}=\left\{n: D x^{n} \equiv 0, n \in \mathbf{N}\right\}-$ the set of $D$-indefinite indices,

$G=G_{d} \cup G_{i n}, \quad N^{*}=F \cup G_{d}$.

(Note that, by the definition of $h$ given previously, we are explicitly including the possibility that $h<0$.)

Then it immediately follows that

Proposition 1. The set $\mathbf{N}$ can be partitioned as

$$
\mathbf{N}=F \cup G=F \cup G_{d} \cup G_{\text {in }}
$$

where $\operatorname{card} G \leq \nu, \operatorname{card} F=\infty$. 
Proof. Considering $D x^{n}$ as a function of $x$ and $n$, we may immediately conclude that

a) $G$ is precisely the subset of the indices that are the natural integer roots of the coefficient of the leading term of $D x^{n}$, which is a polynomial function in $n$, whose degree has as upper bound $\nu$;

b) $G_{\text {in }} \subseteq G$ is the subset of elements of $G$ that are common roots to all coefficients (polynomials in $n$ ) of $D x^{n}$; equivalently, $n \in G_{i n}$ if and only if $\nexists m_{n}$;

c) $\{n: n \in \mathbf{N}, n<-h\} \subset G_{i n}$.

Corollary 1. $G_{d}=\left\{n: m_{n}<n+h, n \in N, n \geq-h\right\}$.

Definiton 2. The degrees $m$ of the polynomials in $D P(x)$ will be called primary if they are of the form $m_{n}$; if not, they will be called derived. The c.p.'s will be called primary or derived, respectively, depending on whether their orders $m$ are primary or derived (since $m$ is the degree of $D Q_{m}(x)$ ). (Primary c.p.'s, as given by a suitable generating formula, will be classified below as generic or singular.)

The determination of the set $M^{*}$ of primary orders is clearly equivalent to the determination of the set $S^{*}=\mathbf{N}-M^{*}, S^{*} \supseteq S$ of the degrees not produced by the action of $D$ on the canonical basis $X$.

Let us now introduce the following definitions:

\section{Definition 3.}

$$
\begin{gathered}
g_{\max }=\max _{g \in G} g, \\
N_{\max }=\left\{n: n \leq g_{\max }, n \in \mathbf{N}\right\}, \\
\bar{N}_{\max }=\left\{n: n<g_{\max }, n \in F\right\}=N_{\max }-G, \\
Z=\left\{n: n \leq g_{\max }+h, n \in \mathbf{N}\right\}, \\
Z_{h}=\{0,1, \ldots, h-1\} \cap \mathbf{N}, \\
Z_{G}=\left\{g_{1}+h, \ldots, g_{\max }+h, g \in G\right\} \cap \mathbf{N}, \\
Z_{n+h}=\left\{n+h, n \in \bar{N}_{\max }\right\} \cap \mathbf{N}, \\
Z_{0}=Z_{h} \cup Z_{G}, Z=Z_{0} \cup Z_{n+h}, \\
m_{G}=\left\{m_{n}, n \in G\right\}, \\
m_{\bar{N}_{\max }}=\left\{m_{n}, n \in \bar{N}_{\max }\right\}=Z_{n+h}, \\
m_{N_{\max }}=\left\{m_{n}, n \in N_{\max }\right\}=m_{G} \cup m_{\bar{N}_{\max }} .
\end{gathered}
$$

Proposition 2. If $G=\phi$, then $S^{*}=Z_{h}$. Thus, in particular, if $h=0$, then $S^{*}=\phi$, so $S=\phi$.

Proof. If $G=\phi$, it follows (Proposition 1) that $N=F$; therefore the application $m$ is defined in $\mathbf{N}$, producing $M^{*}=\left\{m_{n}=n+h, n \in \mathbf{N}\right\}$. But for $\forall n$, it will be $n+h \geq h$ and by definition, $M^{*}=\mathbf{N}-S^{*}$.

(The analogous result for $S$ is already present in Ortiz ([4]).) 
Proposition 3. If $G=G_{\text {in }} \neq \phi$, then $S^{*}=Z_{0}$. Thus, in particular, if $h=0$, it will be $S^{*}=G_{\text {in }}$; if $h<0$ and $g_{\max }<-h$, it will be $S^{*}=\phi$, so $S=\phi$.

Proof. If $G=G_{i n}$, it follows (Proposition 1) that $\mathbf{N}=F \cup G_{i n}$, thus the action $m$ is defined only in $F$, that is, $M^{*}=\left\{m_{n}: m_{n}=n+h, n \in F\right\}$. Following the same reasoning as in the previous proposition, $Z_{h} \subset S^{*}$ and for $g \in G_{i n}$, it will be $(g+h) \notin M^{*}$, and thus also $Z_{G} \subseteq S^{*}$.

Theorem 1. If $G \neq \phi$, then the set $S^{*}$ of the degrees not produced by $D$ acting on the canonical basis satisfies the relation $S^{*}=Z-m_{N_{\max }}$.

Proof. a) If $g_{\max }+h \geq 0$, let us consider $\mathbf{N}-Z=\left\{\ell, \ell>g_{\max }+h, \ell \in \mathbf{N}\right\}$. Then, for $\ell \in \mathbf{N}-Z$, we can always define $n=\ell-h>g_{\max }$, that is, $n \in\left(\mathbf{N}-N_{\max }\right) \subset F$ such that $m_{n}=n+h=\ell$. So $m_{g_{\max }+1}=g_{\max }+h+1$. That is, $(\mathbf{N}-Z)$ is generated by the action $m$ in $\left(\mathbf{N}-N_{\max }\right)$. Thus, $\left.\alpha\right) M^{*}=\mathbf{N}-S^{*} \supseteq \mathbf{N}-Z$, so $\left.S^{*} \subseteq Z ; \beta\right)$ if $\left(Z-S^{*}\right) \neq \phi$, it is produced by the action $m$ in $N_{\max }$. Thus $S^{*}=\left\{n \in Z: n \notin m_{N_{\max }}\right\}$, therefore the proposition is valid.

b) If $g_{\max }+h<0$, then necessarily $h<0$ (note that in the previous case $h \gtreqless 0$ ) and therefore $Z=\phi$. Thus, for $\ell \in \mathbf{N}$, it will be $\ell>g_{\max }+h$, and then, repeating the previous reasoning, it follows that $\mathbf{N}$ is produced by the action $m$ in $\left(\mathbf{N}-N_{\max }\right)$, and consequently: $\left.\alpha) S^{*}=\phi ; \beta\right) m_{N_{\max }}=\phi$. Thus the proposition is also valid.

Corollary 2. If $G \neq \phi$, then the set $S^{*}$ satisfies the relation

$$
S^{*}=Z_{0}-\left[m_{G}-\left(m_{G} \cap Z_{n+h}\right)\right] .
$$

Proof. By definition $Z=Z_{0} \cup Z_{n+h}$. On the other hand, $m_{N_{\max }}=m_{G} \cup Z_{n+h}$. Thus $S^{*}=Z-m_{N_{\max }}=Z_{0}-\left[m_{G}-\left(m_{G} \cap Z_{n+h}\right)\right]$.

Note that Proposition 3 may be immediately (re)-obtained from Theorem 1; Theorem 1 accounts not only for $S^{*}$ but also for the multiplicity structure of the primary c.p.'s, as will be seen later.

Proposition 4. Given $g \in G$, if the degree $(g+h) \in Z$ is produced by the action $m$, that is, $g+h=m \in M^{*}$, then it will have been produced from a singular index $g^{\prime} \in G_{d}$ with $g^{\prime}>g$.

Proof. By hypothesis, let $n \in \mathbf{N}$ such that $m_{n}=n+h$. If $n \in F$ it would be $m_{n}=n+h=g+h$, thus $n \in G$, a contradiction; so $n=g^{\prime} \in G_{d}$, therefore $g+h=m_{g^{\prime}}<g^{\prime}+h$.

Corollary 3. If $G \neq \phi$ and $g_{\max } \geq-h$, then $S^{*} \neq 0$, since at least $\left(g_{\max }+h\right) \in S^{*}$. An analogous result for $h \geq 0$ and $S$, is contained in Ortiz ([4]).

Corollary 4. If $g^{\prime} \in G_{i n}$, with $g^{\prime} \geq-h$ and $g^{\prime}>g^{\prime \prime}$, any $g^{\prime \prime} \in G_{d}$, then $\left(g^{\prime}+h\right) \in$ $S^{*}$.

\section{The indeX-Multiplicity STRUCture of the DifFerential OPERATOR $D$ RElative to THE CANONICAL Basis $X$}

As has been mentioned before, Ortiz has shown that

a) multiplicity in the set of c.p.'s of the linear differential operator $D$, relative to a basis $V$, is an equivalence relation in this set;

b) the quotient set $L$ is uniquely determined by $D$, relative to $V$, and conversely. 
Definition 4. We will refer to $L$ as the multiplicity structure of the c.p.'s of $D$, relative to $V$, and, in particular, to the set $L^{*} \subseteq L$ of the classes of equivalence of multiple primary c.p.'s, as the primary multiplicity structure of $D$, relative to $V$.

Now let us consider, in the indices set $N^{*}$, the equivalence relation $\mu$ associated with the application $m: N^{*} \mapsto M^{*}$, that is $n^{\prime} \mu n^{\prime \prime}$ if and only if $m_{n^{\prime}}=m_{n^{\prime \prime}}$. Then $N^{*}$ is partitioned in $m$-classes of equivalence

$$
M U(m)=\left\{n: m_{n}=m, n \in N^{*}, m \in M^{*}\right\}
$$

whose elements $n$ will be called multiple indices of the degree $m$. The quotient set $M U_{D}=N^{*} / \mu=\left\{M U(m), m \in M^{*}\right\}$ will be called the index-multiplicity structure of the differential operator $D$, relative to the canonical basis $X$. It will further be seen that index-multiplicity is closely related to primary multiplicity $L^{*}$.

When $M U(m)$ has a single element, the index-multiplicity will be said to be trivial; otherwise it will be called effective. We will frequently refer to multiplicity (without qualification) in the strict sense of effective multiplicity.

\section{THE RECURRENCY GENERATING FORMULA FOR THE PRIMARY CANONICAL POLYNOMIALS $\left(S^{*}=S\right)$}

Let us establish the following

Theorem 2. Given the linear differential operator $D$, let $S^{*}$ be the set of degrees not produced by $D$ acting on the canonical basis $X$ (or, equivalently, $M^{*}=\mathbf{N}-S^{*}$, the set of primary degrees of $D)$, and let $M U_{D}=\left\{M U(m), m \in M^{*}\right\}$ be the indexmultiplicity structure determined by $D$.

Then, if $S^{*}=S$ (or, equivalently, $M^{*}=M$ ), the elements $Q_{m}(x)$ of a basis of (primary) canonical polynomials $Q=\left\{Q_{m}(x), m \in M^{*}\right\}$ of $D$, relative to the canonical basis $X=\left\{x^{n}, n \in \mathbf{N}\right\}$, and their multiples with distinct indices, satisfy the recurrency relation

$$
Q_{m}(x)=\frac{1}{a_{m}^{(m)}}\left[x^{n}-\sum_{r \in A_{m}} a_{r}^{(m)} Q_{r}(x)\right],
$$

where each c.p. is defined modulo an arbitrary element $U_{D}(x)$ of the algebraic kernel $U_{D}$ of $D, n \in M U(m)=\left\{n: m_{n}=m, n \in N^{*}\right\}, D x^{n}=\sum_{r=0}^{m} a_{r}^{(m)} x^{r}$ and $A_{m}=\{r \in M: r<m\}$.

Proof. The demonstration is formally analogous to the proof of Ortiz's formula for generating the primary generic c.p.'s. For the conceptual differences between them, we refer the reader to [4] and to the Appendix at the end of this article.

Definition 5. When convenient, we will identify a primary c.p., given by the generating formula, by $Q_{m n}(x)$, it being understood that $n \in M U(m)$; then $n$ is called index of the primary c.p. $Q_{m n}(x)$ of order $m$.

Therefore the recurrence formula may assume the form

$$
Q_{m n}(x)=\frac{1}{a_{m}^{(m n)}}\left[x^{n}-\sum_{r \in A_{m}} a_{r}^{(m n)} Q_{r t}(x)\right], \quad t \in M U(r),
$$

which makes more explicit the fact that, for $S^{*}=S$, the formula generates, in a recurrent way, not only a basis of primary c.p.'s but also all the primary c.p.'s that are their multiples of distinct indices. 
Definition 6. The primary c.p.'s $Q_{m n}(x)$ so constructed will be said to be generic or singular, depending on whether the index $n$ is generic or singular.

Corollary 5. Multiple indices of degree $m$ determine primary c.p.'s, multiples of distinct indices, of order $m, \forall m \in M^{*}$.

The corollary is contained in the statement of the theorem itself.

As we have already pointed out, the generating formula of primary c.p.'s is exact only under the condition $S^{*}=S$.

In any case, the validity of the condition $S^{*}=S$ can be verified by observing whether all the differences between all multiple polynomials (generated by the formula) are exact solutions of $D$; if not, then $S^{*} \neq S$ (that is, there exist not only primary c.p.'s but also derived c.p.'s).

We shall see below that the relation between $S^{*}$ and $S$ is closely associated with the index-multiplicity structure $M U_{D}$ of $D$, and that, in particular, if this multiplicity is trivial (that is, there are no multiple primary c.p.'s with distinct indices), then the validity of the condition $S^{*}=S$ may be assured a priori.

\section{The general properties of the primary multiplicity STRUCTURES OF $D$}

It is clear that the primary multiplicity structures $M U_{D}$ and $L^{*}$ play a central role in the determination of the c.p.'s, which leads us to search for their general properties.

Proposition 5. No class of index-multiplicity has more than one generic index.

Proof. Let us consider, for $\forall m \in M^{*}, n^{\prime}, n^{\prime \prime} \in M U(m)$ with $n^{\prime}, n^{\prime \prime} \in F$. Then $m_{n^{\prime}}=m_{n^{\prime \prime}}=n^{\prime}+h=n^{\prime \prime}+h$, therefore $n^{\prime}=n^{\prime \prime}$.

Corollary 6. Primary generic c.p.'s with distinct indices are never multiple.

Corollary 7. If a pair of primary c.p.'s with distinct indices are multiple, at least one of them is singular.

Corollary 8. Among multiple primary c.p.'s, the generic one, when it exists at all, is the one with minimal index.

Corollary 9. Given $Q_{m g}$, where $g=0$, if there is a multiple c.p. $Q_{m n}$, then $n=g^{\prime}, g^{\prime} \in G_{d}$.

Thus the determination of the multiple primary c.p.'s with distinct indices reduces to the determination of the primary c.p.'s which are multiples of the singular c.p.'s, that is, the primary c.p.'s with index $n=g \in G_{d}$; in other words, the multiplicity structures of $D$ relative to $V$ are ultimately determined by the (singular) multiplicity structure $M U S_{D} \subset M U_{D}$ of the singular indices $n \in G_{d}$.

Corollary 10. If $M U S_{D}$ is trivial, then $M U_{D}$ is also trivial, thus $S^{*}=S$. In particular, this is the case if $G_{d}=\phi$ (thus if $G=\phi$ ).

The reciprocal to this corollary is not necessarily true, that is, it may be the case that $S^{*}=S$ and $M U S_{D}$ is not trivial (see, for example, exs. 2,3), so the case of $D$, the c.p.'s of which are all primary, is very common.

Besides, let us observe that, in particular, this corollary extends Prop. 2 to $S=S^{*}=Z_{h}$ and Prop. 3 to $S=S^{*}=Z_{0}$. 
The general situation of the multiplicity of primary c.p.'s is defined by the following.

Theorem 3. Given $g \in G_{d}$ and the primary c.p. $Q_{m g}(x)$, then if there are multiple primary c.p.'s with distinct indices $Q_{m n}(x)$, they will be of the form $Q_{m n}: g_{\max } \geq$ $n \geq m_{g}-h$ and

a) if $\left(m_{g}-h\right)<0, g$ has no generic multiple;

b) if $\left(m_{g}-h\right)$ is generic, then it will be the generic multiple of $g$; in addition, it will be $n=\left(m_{g}-h\right)$;

c) if $\left(m_{g}-h\right)$ is nongeneric (singular or indefinite), then

c1) $\left(m_{g}-h\right)=g^{\prime} \in G$ is not a multiple of $g$;

c2) $g$ has no generic multiple.

Proof. If there is $n \in \mathbf{N}$ such that $m_{n}=m_{g}$, it will be $m_{n}=m_{g} \leq n+h$; therefore

a) let us assume, by hypothesis, that $\exists n \in \mathbf{N}: m_{n}=n+h=m_{g}$; then $n+h<h$, a contradiction;

b) if $\left(m_{g}-h\right) \in F$, it will be $m_{m_{g}-h}=\left(m_{g}-h\right)+h=m_{g}$;

c1) if $\left(m_{g}-h\right) \notin F$, that is $\left(m_{g}-h\right) \in G$, then either $\left(m_{g}-h\right)$ is indefinite or, if it is definite, $m_{m_{g}-h}<\left(m_{g}-h\right)+h$; that is, $m_{m_{g}-h} \neq m_{g}$;

c2) If there is $n \in F$ such that $n$ is a generic multiple of $g$, then $m_{n}=n+h=m_{g}$; therefore $n=m_{g}-h$, a contradiction, since, by hypothesis, $\left(m_{g}-h\right) \notin F$.

Finally, if an index $n$, a multiple of $g$, is such that $n \in G$, then it will be $n \leq g_{\max }$ and $m_{n}=m_{g}<n+h$, thus $n>m_{g}-h$; if $n \in F$, then $n=m_{g}-h$ and from $m_{n}=n+h=m_{g}<g+h$, it follows that $n<g \leq g_{\max }$. Thus the initial assertion stated by the theorem is immediate.

Corollary 11. Multiple primary c.p.'s have necessarily order $m \leq g_{\max }+h-1$ and index $n \leq g_{\max }$.

Corollary 12. The action $m$ induces, by restriction, the action

$$
m_{N_{\max }}: N_{\max } \rightarrow Z
$$

which defines the singular index-multiplicity structure, thus the multiplicity structure of the primary c.p.'s.

As it has been seen, this is why we prefer to operate with the formula $S^{*}=$ $Z-m_{N_{\max }}$ instead of $S^{*}=Z_{0}-\left[m_{G}-\left(m_{G} \cap Z_{n+h}\right)\right]$; the former accounts not only for $S^{*}$, but also for the singular multiplicity structure of $D$.

Let us now see some particular properties of the primary multiplicity of $D$.

Proposition 6. If a primary c.p. is of the form $Q_{(g+h) g}$, with $g \in G, g^{\prime} \in G_{d}$, then it will be $g^{\prime}>g$.

Proof. $m_{g^{\prime}}=g+h<g^{\prime}+h$.

Proposition 7. Given a primary c.p. $Q_{m g}, g \in G_{d}, m \geq h$, if, for $\forall g^{\prime} \in G_{d}$, (with $g^{\prime}<g$ ) it is $\left(g^{\prime}+h\right) \in S^{*}$, then there is a generic c.p. $Q_{m n}(x), n \in F$, that is, a multiple of $Q_{m g}(x)$.

Proof. Since $m \geq h$, it will be $m=n+h, n \in \mathbf{N}$. Let us assume that $n=g^{\prime} \in G$. Then $m=g^{\prime}+h$, thus (Proposition 6) $g^{\prime}<g$, therefore $m=\left(g^{\prime}+h\right) \in S^{*}$, a contradiction. Thus $n \in F$, therefore (Theorem 3) $Q_{m n}(x)$ is generic. 
Corollary 13. Let $Q_{m g}, m \geq h$ and $g \in G_{d}$, be the minimal element of $G$. Thus there is a generic c.p. $Q_{m(m-h)}(x)$, that is, a multiple of $Q_{m g}(x)$.

Proof. Since $m \geq h$, then $(m-h)=n \in \mathbf{N}$, that is, $m=n+h<g+h$; thus $n<g$ and therefore $n \in F$, so $m_{n}=m$.

Corollary 14. Let $Q_{m g}(x), g \in G_{d}$, be the minimal element of $G$ and $h=0$. Then $Q_{m m}(x)$ is generic.

\section{EXAMPLES}

The following examples illustrate the new theoretical developments; they have been solved by using the software GPC ([16], [17]), which implements the new extensions of the Lanczos-Ortiz theory. In particular, applied to Ortiz's examples $(h \geq 0)$ given in $([4])$, his results have been reobtained.

Ex. 1: (Ortiz, [4]): $D y(x)=x^{3} y^{\prime}(x)-2 y(x)=0$

$$
\begin{aligned}
& D x^{n}=n x^{n+2}-2 x^{n} \\
& h=2 ; G=\{0\} ; G_{d}=\{0\} ; G_{i n}=\phi ; F=\mathbf{N}-\{0\} \\
& g_{\max }=0 ; g_{\max }+h=2 ; Z=\{0,1,2\} ; N_{\max }=\{0\} ; m_{N_{\max }}=\{0\} \\
& S^{*}=\{0,1,2\}-\{0\}=\{1,2\}
\end{aligned}
$$

$N_{\max }$ contains a single element, thus (Cor. 12) $M U S_{D}$ and so also $M U_{D}$ is trivial. Then (Cor. 10) $S^{*}=S$ and $U_{D}=\phi$.

Verification: $m_{0}=0 ; m_{0}-h=-2<0$, thus $g=0$ has no generic multiple, thus has no multiple at all, that is, $M U S_{D}$ is trivial.

The generating formula, without any complementary algorithm, gives all the primary c.p.'s:

$$
Q_{0}(x)=-\frac{1}{2} ; \quad Q_{3}(x)=x ; \quad Q_{4}(x)=\frac{1}{2} x^{2} ; \quad Q_{5}(x)=\frac{1}{3}\left(x^{3}+2 x\right) ; \ldots
$$

Ex. 2: $\left(\right.$ Ortiz, (4)): $D y(x)=\left(x^{2}+1\right) y^{\prime \prime}(x)-6 y(x)=0$

$D x^{n}=(n+2)(n-3) x^{n}+n(n-1) x^{n-2}$

$h=0 ; G=\{3\} ; G_{d}=\{3\} ; G_{\text {in }}=\phi ; F=\mathbf{N}-\{3\}$

$g_{\text {max }}=3 ; g_{\max }+h=3 ; Z=\{0,1,2,3\} ; N_{\max }=\{0,1,2,3\} ; m_{N_{\max }}=\{0,1,2\}$

$S^{*}=\{0,1,2,3\}-\{0,1,2\}=\{3\}$

$m_{3}=1=m_{1} ; M U(1)=\{3,1\}, 1 \in F$

Verification: $m_{3}-h=1 \in F$, thus (Theor. 3 ) 1 is the generic multiple of 3 .

$M U S_{D}$ is not trivial

The generating formula, without any complementary algorithm, gives

$Q_{00}(x)=-\frac{1}{6} ; Q_{11}(x)=-\frac{1}{6} x ; Q_{22}(x)=-\frac{1}{12}\left(3 x^{2}+1\right) ; Q_{13}(x)=\frac{1}{6} x^{3} ;$

$Q_{44}(x)=\frac{1}{6}\left(x^{4}+3 x^{2}+1\right) ; \ldots$

$D\left[Q_{11}(x)-Q_{13}(x)\right]=0$, thus $S^{*}=S$

$C\left(x+x^{3}\right) \in U_{D}$

Ex. 3: $D y(x)=y^{I V}(x)+x y^{I I I}(x)-3 y^{I I}(x)=0$

$$
\begin{aligned}
& D x^{n}=n(n-1)(n-2)(n-3) x^{n-4}+n(n-1)(n-5) x^{n-2} \\
& h=-2 ; G=\{0,1,5\} ; G_{d}=\{5\} ; G_{i n}=\{0,1\} ; F=\mathbf{N}-\{0,1,5\} \\
& g_{\max }=5 ; g_{\max }+h=3 ; Z=\{0,1,2,3\} ; N_{\max }=\{0,1,2,3,4,5\} ; \\
& m_{N_{\max }}=\{0,1,2\} \\
& S^{*}=\{0,1,2,3\}-\{0,1,2\}=\{3\} \\
& m_{5}=1=m_{3} ; M U(1)=\{5,3\} ; 3 \in F
\end{aligned}
$$


Verification: $m_{5}-h=3 \in F$, thus 3 is the generic multiple of $5 . M U S_{D}$ is not trivial.

The generating formula gives directly

$$
\begin{aligned}
& Q_{02}(x)=-\frac{1}{6} x^{2} ; Q_{13}(x)=-\frac{1}{12} x^{3} ; Q_{24}(x)=-\frac{1}{12} x^{4} ; Q_{15}(x)=\frac{1}{120} x^{5} ; \ldots \\
& D\left[Q_{13}(x)-Q_{15}(x)\right]=0, \text { thus } S^{*}=S \\
& {\left[C_{1}\left(10 x^{3}+x^{5}\right)+C_{2} x+C_{3}\right] \in U_{D}}
\end{aligned}
$$

\section{Concluding Remarks}

The inclusion of the differential operators $D$ with negative height extends the Lanczos-Ortiz theory of c.p.'s, Ortiz's recursive process and the theoretical development here presented, to all linear operators $D$ which act on the space of polynomials producing an image with finite co-dimension.

The classification of c.p.'s and of the associated indices, respectively, has allowed us to characterize and focus the case of $D$ the c.p.'s of which are all primary, a very comprehensive case and, at the same time, a case treated with great simplicity by the theoretical construction here elaborated and its computational counterpart, the GPC program; this is well illustrated by the examples that are solved here.

It will be shown elsewhere that the present theoretical scheme and its software can be entirely incorporated, as a first stage, to the theoretical and computational solution of the general case, where $D$ also has derived c.p.'s.

The entire theoretical construction elaborated on here has represented the action of $D$ relative to the canonical basis, but it is clear from Ortiz's works that the entire scheme can be rewritten for any complete polynomial basis.

\section{APPENDIX \\ PROOF OF THE GENERATING FORMULA FOR THE PRIMARY CANONICAL POLYNOMIALS}

Let $M^{*}=\mathbf{N}-S^{*}$ be the set of the primary orders $m \in M^{*}, S^{*}$ having previously been determined on the basis of Theorem 1, and let us assume that $M^{*}=M$ (or $\left.S^{*}=S\right)$, so that all the c.p.'s are primary. Then, for $\forall m \in M, \exists n \in N^{*}$ such that $D x^{n}=m$, therefore $M U(m) \neq 0$, and $n \in M U(m)$, that is, $n$ is any one of the multiple indices of degree $m$ and

$$
D x^{n}=\sum_{r=0}^{m} a_{r}^{(m n)} x^{r}, \quad n \in M U(m) .
$$

(Note that the consideration of $D x^{n}$ with $n \in M U(m)$ implies at once that $a_{m}^{(m n)} \neq$ 0$.

Now, let there be a polynomial defined by

$$
T_{m n}(x)=\frac{1}{a_{m}^{(m n)}}\left[x^{n}-\sum_{r \in A_{m}} a_{r}^{(m n)} Q_{r t}(x)\right],
$$

where

$$
A_{m}=\{r \in M, r<m\}, \quad t \in M U(r) .
$$

Hence

$$
D T_{m n}(x)=x^{m}+\frac{1}{a_{m}^{(m n)}}\left[\sum_{r=0}^{m-1} a_{r}^{(m n)} x^{r}-\sum_{r \in A_{m}} a_{r}^{(m n)}\left(x^{r}+R_{r t}(x)\right)\right],
$$


where $R_{r t}(x) \in R(x)$. Then, naming $S_{m}=\{r \in S, r<m\}$, it will be

$$
\begin{aligned}
D T_{m n}(x) & =x^{m}+\frac{1}{a_{m}^{(m n)}}\left[\sum_{r \in S_{m}} a_{r}^{(m n)} x^{r}-\sum_{r \in A_{m}} a_{r}^{(m n)} R_{r t}(x)\right] \\
& =x^{m}+R_{m n}(x),
\end{aligned}
$$

where $R_{m n}(x) \in R(x)$.

Thus $T_{m n}(x)$ is a primary c.p. $Q_{m n}(x) \equiv T_{m n}(x)$, either generic or singular, depending on the index $n$, and the above formula in fact generates, in a recurrent way, bases of primary c.p.'s and also all their multiple c.p.'s (with distinct indices). Clearly, any $T_{m n}(x)+U_{D}(x)$, with $U_{D}(x) \in U_{D}$, will be a (primary) c.p. multiple of $T_{m n}(x)$.

\section{ACKNOWLEDGEMENTS}

Thanks are due to Prof. Therezinha Costa (PUC-Rio), who gave valuable suggestions for this work and called our attention to the basic articles by Ortiz and Ortiz and Chaves.

The author is greatly indebted to Prof. E.L. Ortiz's papers on the subject.

The author would also like to thank Prof. Maria Lucia Scerni Barbosa for her participation in a preliminary study of Ortiz's article [4]. Thanks are also due to Prof. D.A. Hill for useful comments and to Prof. J.L.M. Rangel Netto for friendly encouragement.

The author wishes to thank the Conselho Nacional de Desenvolvimento Científico e Tecnológico (C.N.Pq.) in Brazil for its support during the preparation of this paper.

\section{BIBLIOGRAPHY}

[1] Lanczos, C.: Trigonometric interpolation of empirical and analytical functions, J. Math. Phys. 17, (1938), 123-199.

[2] Lanczos, C.: "Applied Analysis", Prentice Hall, New Jersey, (1956). MR 18:823c

[3] Llorente, P., Ortiz, E. L.: Sur quelques aspects algébriques d'une méthode d'approximation de M. Lanczos, Math. Notae, 21, (1968), 17-23. MR 39:7823

[4] Ortiz, E.L.: The Tau Method, SIAM J. Numer. Anal. 6, (1969), 480-491. MR 41:2934

[5] Ortiz, E.L.: "Canonical polynomials in Lanczos Tau Method", in Studies in Numerical Analysis (B.P.K. Scaife, Ed.), Academic Press, London, (1974). MR 57:14478

[6] Chaves, T. (Costa, Therezinha S.), Ortiz, E.L.: On the numerical solution of two point boundary valued problems for linear differential equations, Z. Angew Math. Mech., 48, (1968), 415-418. MR 39:7824

[7] Ortiz, E.L.: On the numerical solution of non-linear and functional-differential equations with the Tau Method, in Numerical Treatment of Differential Equations in Applications (Ansorg, R., Törnig, W. eds.), 127-139, Berlin, Springer-Verlag (1978). MR 80c:65180

[8] Namasivayam, S., Ortiz, E.L.: Best approximation and the numerical solution of partial differential equations with the Tau Method, Portugaliae Mathematica 40, (1981), 97-119. MR 88a:65118

[9] Liu, K.M., Ortiz, E.L.: Numerical solution of ordinary and partial functional-differential eigenvalue problems with the Tau Method, Computing 41, (1989), 205-217. MR 90c:65156

[10] Liu, K.M., Ortiz E.L.: Numerical solution of eigenvalue problems for partial differential equations with the Tau-lines Method, Comp. and Maths. with Appls. 12B, (1986), 11531168. MR 88e: 65130

[11] Ortiz, E.L., Samara, H.: An operational approach to the Tau Method for the numerical solution of non-linear differential equations, Computing 27, (1981), 15-25. MR 83b:65079

[12] Freilich, J.H., Ortiz E.L.: Numerical solution of systems of ordinary differential equations with the Tau Method: An error analysis, Math. Comp., 39, (1982), 467-479. MR 84k:65066 
[13] Hosseini, M., Abadi Ali, Ortiz, E.L.: A Tau Method based on non-uniform space-time elements for the numerical simulation of solutions, Computers Math. Applic., vol. 22, No 9 (1991), 7-19.

[14] Khajah, H.G., Ortiz E.L.: Numerical approximation of solutions of functional equations using the Tau Method, Applied Numerical Mathematics 9 (1992), 461-474. MR 92k:65187

[15] El-Daou, M.K., Ortiz, E.L., Samara, H.: A unified approach to the Tau Method and Chebyshev series expansion techniques, Computers Math. Applic., vol. 25, No 3 (1993), 73-82. MR 94b:65106

[16] Bunchaft, M.E. Froes: GPC (Gera Polinômios Canônicos) Programa em linguagem Fortran, Projeto Final de Programação, P.U.C. (1994).

[17] Bunchaft, M.E. Froes, Costa, Therezinha S.: Software for a method of determination of a basis of primary canonical polynomials in the Tau Method (to be submitted for publication in Appl. Math. Modelling).

Departamento de Ciências da ComputaÇão, Instituto de Matemática, Universidade Federal da Bahia, Salvador (Bahia), Brasil

E-mail address: bunchaft@ufba.br 